\title{
Minimal energy for elastic inclusions
}

\author{
By Hans KnÜpfer and Robert V. Kohn \\ Courant Institute, New York University \\ Mercer Street 251, New York, NY 10012, USA
}

We consider a variant of the isoperimetric problem with a nonlocal term representing elastic energy. More precisely, our aim is to analyse the optimal energy of an inclusion of fixed volume whose energy is determined by surface and elastic energy. This problem has been studied extensively in the physical/metallurgical literature; however, the analysis has mainly been either (i) numerical, or (ii) restricted to a specific set of inclusion shapes, e.g. ellipsoids. In this article we prove a lower bound for the energy, with no a priori hypothesis on the shape (or even number) of the inclusions.

Keywords: linear elasticity, precipitate, phase transformation

\section{Introduction}

We consider a variant of the isoperimetric problem with a nonlocal term representing elastic energy. More precisely, our aim is to analyse the optimal energy of an inclusion of fixed volume whose energy is the sum of surface and elastic energy. We note that this problem has been studied extensively in the physical/metallurgical literature. However, in this literature, the analysis has mainly been either (i) numerical, or (ii) restricted to a specific set of inclusion shapes, e.g. ellipsoids. Such studies give upper bounds on the minimum energy. In this article we prove a corresponding lower bound, with no a priori hypothesis on the shape (or even number) of the inclusions.

Elastic inclusions can be observed when a material undergoes a phase transformation between two preferred strains, which may e.g. be elicited by a change of temperature. In this case, the phase transformation is initiated by the creation and growth of a small nucleus representing the new material state. The saddle point between the two uniform phases is represented by the critical nucleus whose energy describes the energy barrier between the two uniform phases.

In the classical theory of nucleation, the size and shape of the critical nucleus is determined by a competition between bulk energy and interfacial energy. While the bulk energy favours the emergence of the new phase, the interfacial energy provides an energy barrier for the creation and growth of the nucleus. In this situation where all the terms contributing to the energy are local, the optimal shape of the inclusion does not depend on its size. The minimisers are well known: In the simplest situation when the interfacial energy is isotropic, the shape of the inclusion is a sphere. More generally, when the interfacial energy is anisotropic, the minimisers take the form of the well-known Wulff-shape, see e.g. (Wulff 1901, Taylor 1975, Fonseca \& Müller 1991).

The elastic energy introduces a length scale into the problem; in particular, the shape of the optimal inclusion depends on its volume. The energy of inclusions in 
the presence of elastic energy has mainly been studied numerically or assuming an ellipsoidal inclusion shape. Numerical simulations for the morphology and evolution of the nucleus have been given e.g. by Voorhees et al.(1992) and Zhang et al. (2007, 2008). In the physical literature, the shape (and the growth) of the inclusion has been optimized within an ansatz, see e.g. Khachaturyan (1982), Mura (1982), Brener et al. (1999) and Wang \& Khachaturyan (1994). While such calculations give much insight, they only apply for certain restricted classes of configurations.

Early work on mathematical analysis of energy-driven pattern formation includes the analysis by Kohn \& Müller $(1992,1994)$ of a toy model in elasticity theory. Related problems have attracted increasing attention in past years with the analysis of various models (Choksi \& Kohn 1998, Choksi et al. 1999, Alberti et al. 2009, Capella \& Otto 2009, 2010). While most of the previous analysis does not address the dependence of the energy on the volume fraction of the different phases, recently the case of extreme volume fraction has gained more attention: In (Choksi et al. 2008), the intermediate state of a type-I superconductor is studied by ansatz-free analysis for the case of extreme volume fraction. Another recent result addresses the energy scaling in a bulk ferromagnet in the presence of an external field of critical strength (Knüpfer \& Muratov 2010). In all of the above models, the energy is characterized by a nonlocal term and a local, regularizing term of higher order. One special feature of the elastic energy is the fact that the nonlocal term is anisotropic. Insightful analysis has been developed on determining possible configurations that are free of elastic energy, see e.g. (Dolzmann \& Müller 1995, Müller \& Sverak 1996).

Our main result, theorem 2.1, is an ansatz-free lower bound for the energy when the volume of the inclusion phase is fixed. Let us give an overview of the arguments that are used in the proof. There are two main ingredients: The first ingredient is a covering argument which reduces the task to a local problem by identifying a local length scale where elastic and interfacial energy are in balance. The second main ingredient is a lower bound for the elastic energy for the local problem. Here, the analysis takes advantage of the discreteness of the phase function $\chi$ which describes the shape of the minority phase. In a related context, discreteness of $\chi$ has been used to prove rigidity results or to give lower bounds on the energy (Dolzmann \& Müller 1995, Capella \& Otto 2009). While most of the mathematical arguments in this article are based on the sharp-interface description of the elastic energy, we also present the two examples of diffuse-interface models and show how our results extend to these models.

Structure of the article. In $\S 2$, we introduce the model and state our results for both the sharp and diffuse-interface models. In $\S 3$, we give the proof of our result for the sharp-interface model. In $\S 4$, we discuss the diffuse-interface models. Basic results about geometrically linear elasticity are collected in the appendix.

Notation. The following notation will be used throughout the article: By a universal constant, we mean a constant that only depends on the dimension $d$. The symbols $\sim, \lesssim$ and $\gtrsim$ indicate that an estimate holds up to a universal constant. For example, $A \sim B$ says that there are universal constants $c, C>0$ such that $c A \leq B \leq C A$. The symbols $\ll$ and $\gg$ indicate that an estimate requires a small universal constant. If we e.g. say that $A \lesssim B$ for $\epsilon \ll 1$, this means that $A \leq C B$ holds for all $\epsilon \leq \epsilon_{0}$ where $\epsilon_{0}>0$ is a small universal constant.

The mean value of a function $f$ on the set $E$ is denoted by $\langle f\rangle_{E}$. For $u \in B V(E)$, 
the total variation of $u$ is sometimes denoted by $\|D u\|_{E}$. The Fourier transform of $u$ is defined by $\hat{u}(\xi)=(2 \pi)^{-d / 2} \int e^{i \xi \cdot x} u(x) d x$, in particular Parseval's identity holds with constant 1 . The set of $d \times d$ matrices is denoted by $\mathcal{M}(d)$, the set of symmetric matrices by $\mathcal{S}(d) \subset \mathcal{M}(d)$. The set of 'compatible strains' $\mathcal{V}(d) \subset \mathcal{S}(d)$ is

$$
\mathcal{V}(d)=\left\{A \in \mathcal{S}(d): A=\frac{1}{2}(u \otimes v+v \otimes u) \text { for some } u, v \in \mathbb{R}^{d}\right\},
$$

where the tensor product $u \otimes v \in \mathcal{M}(d)$ is defined componentwise by $(u \otimes v)_{i j}=u_{i} v_{j}$. Finally, for $A, B \in \mathcal{M}(d)$, the contraction is defined by $A: B=\sum_{i, j} A_{i j} B_{i j}$, and the corresponding matrix norm is given by $\|A\|=\sqrt{A: A}$.

\section{Model and statement of results}

In the framework of geometrically nonlinear elasticity, the energy associated to the deformation $y: \Omega \rightarrow \mathbb{R}^{d}$ of an elastic body with domain of reference $\Omega \subseteq \mathbb{R}^{d}, d \geq 2$, is given by

$$
\int_{\Omega} \mathcal{W}(\nabla y) d x
$$

where the nonconvex energy density $\mathcal{W}$ describes the preferred states of the material, see e.g. (Ball \& James 1987, Bhattacharya 2003). Each preferred crystal configuration of the material corresponds to a well of the elastic energy density. We want to analyse the case where the elastic energy has two preferred phases. Furthermore, we use the geometrically linear approximation of the nonlinear theory (2.1) studied e.g. by Khachaturyan (1967) and Roitburd (1969), see also (Kohn 1991, Bhattacharya 1993). In this theory, the deformation is described by the displacement $u(x)=y(x)-x$ while the elastic energy is a function of the linear strain $e(u)=\frac{1}{2}\left(\nabla u+\nabla^{t} u\right)$. Also, it is well accepted that on small scales the energy should be complemented by a higher order term. Therefore, we include a sharp-interface energy penalizing the interfaces between the two phases. Finally, we include a term that captures the energetic favorability of the new phase.

The above considerations motivate considering the energy

$$
\mathcal{E}(\chi, u)=\eta \int_{\mathbb{R}^{d}}|\nabla \chi|+\int_{\mathbb{R}^{d}}\|e(u)-\chi F\|^{2}-\gamma \int_{\mathbb{R}^{d}} \chi,
$$

where $F \in \mathcal{S}(d)$. In this model, the two preferred phases are represented by $0, F$. The elastic energy $\|e(u)-\chi F\|^{2}$ uses a trivial Hooke's Law; this represents no loss of generality since we seek a lower bound and our focus lies on its scaling law not the prefactor. The characteristic function $\chi \in B V\left(\mathbb{R}^{d},\{0,1\}\right)$ describes the region occupied by the minority phase, see also Chapter 12 in Bhattacharya (2003). It allows us to define the volume of the inclusion

$$
\mu=\int_{\mathbb{R}^{d}} \chi
$$

The set $\mathcal{A}(\mu)$ of admissible functions for prescribed volume $\mu$ is given by

$$
\mathcal{A}(\mu):=\left\{(\chi, u) \in B V\left(\mathbb{R}^{d},\{0,1\}\right) \times H^{1}\left(\mathbb{R}^{d}, \mathbb{R}^{d}\right): \chi \operatorname{satisfies}(2.3)\right\} .
$$


Theorem 2.1 (Scaling of energy). Let $e(\mu):=\inf _{(\chi, u) \in \mathcal{A}(\mu)} \mathcal{E}(\chi, u)$, and define $\delta:=\inf _{P \in \mathcal{V}(d)}\|F-P\|^{2}$ where $\mathcal{V}(d)$ is the set of compatible strains defined in (1.1). In any dimension $d \geq 2$ we have the upper bound

$$
e(\mu)+(\gamma-\delta) \mu \lesssim \max \left\{\eta \mu^{\frac{d-1}{d}}, \eta^{\frac{d}{2 d-1}}\|F\|^{\frac{2 d-2}{2 d-1}} \mu^{\frac{2 d-2}{2 d-1}}\right\}
$$

and the lower bounds

$$
\begin{aligned}
e(\mu)+(\gamma-\delta) \mu & \gtrsim \eta \mu^{\frac{d-1}{d}} \\
e(\mu)+\gamma \mu & \gtrsim \eta^{\frac{d}{2 d-1}}\|F\|^{\frac{2 d-2}{2 d-1}} \mu^{\frac{2 d-2}{2 d-1}} .
\end{aligned}
$$

In particular (averaging the two lower bounds) we have

$$
e(\mu)+\gamma \mu \sim \delta \mu+ \begin{cases}\eta \mu^{\frac{d-1}{d}} & \text { if } \mu \leq \eta^{d}\|F\|^{-2 d}, \\ \eta^{\frac{d}{2 d-1}}\|F\|^{\frac{2 d-2}{2 d-1}} \mu^{\frac{2 d-2}{2 d-1}} & \text { if } \mu \geq \eta^{d}\|F\|^{-2 d},\end{cases}
$$

Equation (2.7) gives the scaling of the minimal energy up to a universal constant. The first term on the right, $\delta \mu$, is related to incompatibility of the two phases; it vanishes if they are elastically compatible. The second term is independent of the compatibility between the two phases but depends on the magnitude of the "eigenstrain" $F$. For small inclusions, interfacial energy is dominant and the optimal scaling is achieved by a sphere. On the other hand, for larger inclusions, the shape of the minimiser is determined by competition between elastic energy and interfacial energy. In this case the optimal scaling is achieved by an inclusion with shape of a thin disc, whose large surfaces lie in the twin planes between the two phases. Equation (2.7) shows that this well-known picture is energetically optimal.

When $\eta \rightarrow 0$ with all other parameters held fixed, (2.4) and (2.5) show that

$$
\lim _{\eta \rightarrow 0} e(\mu)=(\delta-\gamma) \mu
$$

Thus for $\eta>0$ we have

$$
e(\mu)=(\delta-\gamma) \mu+\text { correction due to positive } \eta .
$$

In view of (2.4), it is natural to guess that the correction defined by (2.8) is of order $\max \left\{\eta \mu^{\frac{d-1}{d}}, \eta^{\frac{d}{2 d-1}}\|F\|^{\frac{2 d-2}{2 d-1}} \mu^{\frac{2 d-2}{2 d-1}}\right\}$. However our results fall short of proving this, since the left hand side of $(2.6)$ is $e(\mu)+\gamma \mu$ rather than $e(\mu)+(\gamma-\delta) \mu$. (For a brief discussion why, see the end of $\S 3$.)

The energy barrier between the two uniform states in model (2.2) can be calculated by a minimax principle: first, identify the global minimum $e(\mu)$ of all configurations with fixed volume $\mu$; then identify the maximum $\max _{\mu>0} e(\mu)$ over all $\mu>0$. The corresponding minimizing configuration with this volume is the critical nucleus for the phase transformation. Its energy describes the energy barrier between the two uniform phases, in the absence of defects or boundaries.

As a consequence of theorem 2.1, we identify three regimes that characterize the energy scaling and the size of the critical nucleus for $(2.2)$ :

Theorem 2.2 (Critical nucleus). Let $e(\mu)$ and $\delta$ be as in Theorem 2.1. Then 
1. For $\gamma \ll \delta$, we have $\max _{\mu>0} e(\mu)=\infty$ and $\operatorname{argmax}_{\mu>0} e(\mu)=\infty$.

2. For $\gamma \gg \delta$ and $\gamma \ll\|F\|^{2}$, we have $\max _{\mu>0} e(\mu) \sim \eta^{d} \gamma^{2-2 d}\|F\|^{2 d-2}$ and $\mu^{*} \sim \eta^{d} \gamma^{1-2 d}\|F\|^{2 d-2}$ for all $\mu^{*} \in \operatorname{argmax}_{\mu>0} e(\mu)$.

3. For $\gamma \gg \delta$ and $\gamma \gg\|F\|^{2}$, we have $\max _{\mu>0} e(\mu) \sim \eta^{d} \gamma^{1-d}$ and $\mu^{*} \sim \eta^{d} \gamma^{-d}$ for all $\mu^{*} \in \operatorname{argmax}_{\mu>0} e(\mu)$.

Theorem 2.2 is a direct consequence of (2.7). In the first of its three regimes the energy barrier is infinite, so there is no critical nucleus of finite size. This excludes a phase transformation in an infinite sample. Note that the first regime can only occur for an incompatible inclusion. In the second and third regimes the size of the critical nucleus is finite. Depending on the relative size of $\gamma$ and $\|F\|$, the scaling and the size of the critical nucleus is however quite different. In particular, the second regime corresponds to a penny-shaped nucleus while the third regime corresponds to an (approximately) spherical nucleus. The cases in Theorem 2.2 do not cover all possible values of the parameters (for example, they do not cover the case $\gamma=\delta$ ). This is because we don't have a lower bound directly analogous to (2.4).

We note that the physical relevance of theorem 2.2 relies on the following physical assumptions about the phase transformation: 1) The volume $\mu$ is supposed to be a continuous function in time. 2) For fixed time $t$ and volume $\mu(t)$, the configuration achieves the optimal shape. 3) Finite-size effects (such as nucleation at a boundary or corner) are being ignored. 4) The critical nucleus has a reasonably sharp interface.

Theorems 2.1 and 2.2 address the sharp-interface energy (2.2), but the same ideas can also be used in a diffuse-interface setting. We shall explain this in $\S 4$ where we define two diffuse-interface analogies of $(2.2), \tilde{\mathcal{E}}_{1}$ and $\tilde{\mathcal{E}}_{2}$. Their minimum-energy scaling laws are the same as that of $\mathcal{E}$ :

Theorem 2.3 (Diffuse-interface energies). For $\gamma=0$, we have

$$
\inf _{(\chi, u) \in \mathcal{A}(\mu)} \mathcal{E}(\chi, u) \sim \inf _{u \in \tilde{\mathcal{A}}_{1}(\mu)} \tilde{\mathcal{E}}_{1}(u) \sim \inf _{(\tilde{\chi}, u) \in \tilde{\mathcal{A}}_{2}(\mu)} \tilde{\mathcal{E}}_{2}(\tilde{\chi}, u) .
$$

We remark that our estimates only capture the scaling but not the leading order constant of the minimal energy. Consequently, the results do not give information about the precise shape of the minimisers. While we expect that when $\mu$ is large the minimiser resembles a thin disc, this does not follow from our analysis. However, our analysis shows that a thin disc (with an appropriate ratio of height and diameter) is optimal in terms of the scaling of the energy. We expect that a more precise estimate of the shape of the optimal inclusion would require the use of the Euler-Lagrange equation for (2.2).

\section{Proof of theorem 2.1}

In $\S 3 \mathrm{a}$, we give two lower bounds on the elastic energy. The proof of theorem 2.1 is then given in $\S 3 \mathrm{~b}$.

(a) Lower bounds for the elastic energy

In this section, we give two lower bounds on the elastic energy in propositions 3.1 and 3.5. The main result is the following lower bound for the elastic energy: 
Proposition 3.1. Let $d \geq 2$ and let $\chi \in B V\left(B_{R},\{0,1\}\right)$. There exist constants $c_{d}$ and $\alpha_{d}$ such that if

$$
\|\chi\|_{L^{1}\left(B_{R}\right)} \leq c_{d} R^{d} \quad \text { and } \quad\|\nabla \chi\|_{B_{R}} \leq c_{d} R^{d-1},
$$

then we have

$$
\inf _{u \in H^{1}\left(\mathbb{R}^{d}, \mathbb{R}^{d}\right)}\|e(u)-\chi F\|_{L^{2}\left(B_{R}\right)}^{2} \geq c_{d} R^{-d}\|F\|^{2}\|\chi\|_{L^{1}\left(B_{\alpha_{d} R}\right)}^{2} .
$$

Although this estimate does not assume compatibility of $F$, it is most relevant in this case: The case of incompatible $F$ leads to a higher elastic energy and is treated in proposition 3.5. The two conditions in (3.1) state that the volume fraction of the minority phase should be relatively small in $B_{R}$ and that the interfacial energy should be small compared to $\partial B_{R}$. Both conditions in (3.1) are necessary: If we did not assume $\|\chi\|_{L^{1}\left(B_{R}\right)} \ll 1$, then the configuration with uniform gradient $F$ would not cost any elastic energy and hence would yield a counter example. The second condition in (3.1) excludes stripe like patterns where the stripes are aligned with the twin planes between the strains $F$ and 0 . Such laminar structures also would not yield any contribution of elastic energy (if $F$ is compatible).

We split the proof of proposition 3.1 into two parts. We first prove the case $d=2$ in lemma 3.2 , before turning to the case of general $d \geq 2$ in lemma 3.4. The proof for $d=2$ relies strongly on the fact that the interfacial energy is discrete on the boundary of two-dimensional sets. Since compatibility is a two-dimensional issue (see also lemma 4.2), it is not surprising that the case $d=2$ is special.

Lemma 3.2. Proposition 3.1 holds for $d=2$ and $\alpha_{2}=1 / 5$.

Proof. By the rescaling $x \mapsto x / R$ and by a rotation, it is enough to consider $R=1$ and $F=\operatorname{diag}\left(\lambda_{1}, \lambda_{2}\right)$. Furthermore, without loss generality, we assume $\lambda_{1} \geq\left|\lambda_{2}\right|$. We argue by contradiction and assume that (3.2) does not hold; i.e. for some fixed universal but arbitrarily small constant $c_{2}$, we have

$$
\left\|\partial_{1} u_{1}-\lambda_{1} \chi\right\|_{L^{2}\left(B_{1}\right)}+\left\|\partial_{2} u_{2}-\lambda_{2} \chi\right\|_{L^{2}\left(B_{1}\right)}+\left\|\partial_{2} u_{1}+\partial_{1} u_{2}\right\|_{L^{2}\left(B_{1}\right)} \leq c_{2} \lambda_{1} \mu,
$$

where we have set $\mu:=\|\chi\|_{L^{1}\left(B_{\alpha}\right)}$ (Here and below we write $\alpha$ in place of $\alpha_{2}$ for simplicity of notation). In the following, we will not keep track about the precise form of the constants. Instead, we use the notation $\ll$ if an estimate holds for a universal but small constant. We thus write (3.3) as

$$
\left\|\partial_{1} u_{1}-\lambda_{1} \chi\right\|_{L^{2}\left(B_{1}\right)}+\left\|\partial_{2} u_{2}-\lambda_{2} \chi\right\|_{L^{2}\left(B_{1}\right)}+\left\|\partial_{2} u_{1}+\partial_{1} u_{2}\right\|_{L^{2}\left(B_{1}\right)} \ll \lambda_{1} \mu .
$$

Step 1: Notation and choice of $Q^{(i)}$. It is more convenient to work with rectangles instead of balls. We cut out three rectangles $Q^{(i)}=I_{1}^{(i)} \times I_{2} \subset B_{3 \alpha}$, $i=1,2,3$, where $I_{1}^{(i)}=\left[x_{1}^{(i-1)}, x_{1}^{(i)}\right]$ with $x_{1}^{(i-1)}<x_{1}^{(i)}$, see figure 1 . Also let $I_{1}:=\bigcup_{i} I_{1}^{(i)}$. We choose the sets such that $B_{\alpha} \subset Q^{(2)}$, in particular $\int_{Q^{(2)}} \chi \geq$ $\mu$. Furthermore, we may assume that the side lengths of $Q^{(i)}$ are of order 1, i.e. $\left|I_{1}^{(i)}\right| \sim 1,\left|I_{2}\right| \sim 1$ (i.e. up to a universal constant). Note that this is possible for $\alpha=1 / 5$; the argument is not optimized in $\alpha$. By Fubini's theorem and by adjusting the sets slightly, we may also assume that, on the boundaries of the sets $Q^{(i)}$, there 


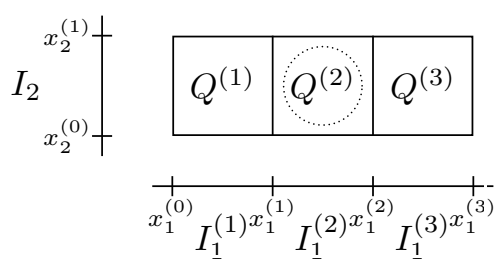

Figure 1. Sketch of the geometry and notations used

is no concentration of energy and no concentration of the minority phase, i.e. the following integrals are well-defined and we have (using (3.1), (3.4))

$$
\begin{aligned}
\|e(u)-\chi F\|_{L^{2}\left(\bigcup_{i=1}^{3} \partial Q^{(i)}\right)} & \lesssim\|e(u)-\chi F\|_{L^{2}\left(B_{1}\right)} \stackrel{(3.4)}{\ll} \lambda_{1} \mu, \\
\|\nabla \chi\|_{\bigcup_{i=1}^{3}} \partial Q^{(i)} & \lesssim\|\nabla \chi\|_{B_{1}} \stackrel{(3.1)}{\ll} 1, \\
\|\chi\|_{L^{1}\left(\bigcup_{i=1}^{3} \partial Q^{(i)}\right)} & \lesssim\|\chi\|_{L^{1}\left(B_{1}\right)} \stackrel{(3.1)}{\ll} 1 .
\end{aligned}
$$

Step 2: Only majority phase on $\partial Q^{(i)}$. By discreteness of the surface energy on lines and discreteness of $\chi,(3.6)-(3.7)$ can be improved. In fact,

$$
\begin{gathered}
\|\nabla \chi\|_{\bigcup_{i=1}^{3} \partial Q^{(i)}}=0, \\
\|\chi\|_{L^{1}\left(\bigcup_{i=1}^{3} \partial Q^{(i)}\right)}=0 .
\end{gathered}
$$

Indeed, since the sets $\partial Q^{(i)}$ are one-dimensional, the measure in (3.6) is discrete; hence (3.6) strengthens to (3.8). Furthermore since $\chi \in\{0,1\}$ and in view of (3.8), (3.7) strengthens to (3.9).

Step 3: Smallness of $u_{1}, u_{2}$ on $\partial Q^{(i)}$. In this step, we show that (after normalization) $u_{1}$ is small on all horizontal boundaries and $u_{2}$ is small on all vertical boundaries of the sets $Q^{(i)}$, i.e.

$$
\left\|u_{1}\right\|_{L^{\infty}\left(I_{1} \times\left\{x_{2}^{(j)}\right\}\right)}+\left\|u_{2}\right\|_{L^{\infty}\left(\left\{x_{1}^{(i)}\right\} \times I_{2}\right)} \ll \lambda_{1} \mu,
$$

where $i=0,1,2,3$ and $j=0,1$. Since the energy only depends $e(u)$, we may transform $u$ by transformations that only affect the anti-symmetric part of $D u$. In particular, by the change of variables

$$
\begin{aligned}
& u_{1} \mapsto u_{1}-\frac{x_{2}-x_{2}^{(0)}}{x_{2}^{(1)}-x_{2}^{(0)}}\left\langle u_{1}\right\rangle_{I_{1} \times\left\{x_{2}^{(1)}\right\}}-\frac{x_{2}^{(1)}-x_{2}}{x_{2}^{(1)}-x_{2}^{(0)}}\left\langle u_{1}\right\rangle_{I_{1} \times\left\{x_{2}^{(0)}\right\}}, \\
& u_{2} \mapsto u_{2}+\frac{x_{1}}{x_{2}^{(1)}-x_{2}^{(0)}}\left\langle u_{1}\right\rangle_{I_{1} \times\left\{x_{2}^{(1)}\right\}}-\frac{x_{1}}{x_{2}^{(1)}-x_{2}^{(0)}}\left\langle u_{1}\right\rangle_{I_{1} \times\left\{x_{2}^{(0)}\right\}},
\end{aligned}
$$

we may assume that the average of $u_{1}$ vanishes on both horizontal boundaries, i.e.

$$
\left\langle u_{1}\right\rangle_{I_{1} \times\left\{x_{2}^{(j)}\right\}}=0, \quad \text { for } j=0,1 .
$$

Similarly, by the change of variables $u_{2} \mapsto u_{2}-\left\langle u_{2}\right\rangle_{\left\{x_{1}^{(0)}\right\} \times I_{2}}$, we may also assume that the average of $u_{2}$ vanishes on the left vertical boundary, i.e. $\left\langle u_{2}\right\rangle_{\left\{x_{1}^{(0)}\right\} \times I_{2}}=0$. 
By (3.9), we now obtain $L^{\infty}$ - control of $u_{1}$ on both horizontal boundaries, i.e.

$$
\left\|u_{1}\right\|_{L^{\infty}\left(I_{1} \times\left\{x_{2}^{(j)}\right\}\right)} \stackrel{(3.11)}{\lesssim}\left\|\partial_{1} u_{1}\right\|_{L^{2}\left(I_{1} \times\left\{x_{2}^{(j)}\right\}\right)} \stackrel{(3.5)}{\ll} \lambda_{1} \mu, \quad \text { for } j=0,1 .
$$

In particular, our supremum control of $u_{1}$ on the horizontal boundaries yields

$$
\left|\left\langle\partial_{2} u_{1}\right\rangle_{Q^{(i)}}\right| \stackrel{(3.12)}{\ll} \lambda_{1} \mu, \quad \text { for } i=1,2,3 .
$$

We next use the cross-diagonal part of the energy to get similar control for $u_{2}$ on all vertical boundaries of $Q^{(i)}$. For this, we note that by Jensen's inequality, we have

$$
\left|\left\langle\partial_{2} u_{1}+\partial_{1} u_{2}\right\rangle_{Q^{(i)}}\right| \lesssim\left\|\partial_{2} u_{1}+\partial_{1} u_{2}\right\|_{L^{2}\left(Q^{(i)}\right)} \stackrel{(3.4)}{\ll} \lambda_{1} \mu, \quad \text { for } i=1,2,3 .
$$

Estimates (3.13) and (3.14) together yield $\left|\left\langle\partial_{1} u_{2}\right\rangle_{Q^{(i)}}\right| \ll \lambda_{1} \mu$ for $i=1,2$, 3. Together with our normalization, this shows that $u_{2}$ is small in average on all vertical boundaries of $Q^{(i)}$, i.e.

$$
\left|\left\langle u_{2}\right\rangle_{\left\{x_{1}^{(i)}\right\} \times I_{2}}\right| \ll \lambda_{1} \mu, \quad \text { for } i=1,2,3 .
$$

By (3.5) and (3.9), we then get control on $u_{2}$ on all vertical boundaries of $Q^{(i)}$, i.e.

$$
\left\|u_{2}\right\|_{L^{\infty}\left(\left\{x_{1}^{(i)}\right\} \times I_{2}\right)} \lesssim\left|\left\langle u_{2}\right\rangle_{\left\{x_{1}^{(i)}\right\} \times I_{2}}\right|+\left\|\partial_{2} u_{2}\right\|_{L^{2}\left(\left\{x_{1}^{(i)}\right\} \times I_{2}\right)} \stackrel{(3.15),(3.4)}{\ll} \lambda_{1} \mu,
$$

for $i=0,1,2,3$. This concludes the proof of $(3.10)$.

Step 4: Smallness of $u_{1}, u_{2}$ in $Q^{(i)}$. In this step, we show that $u_{1}$ is small in average in all $Q^{(i)}$, i.e.

$$
\left\langle u_{1}\right\rangle_{Q^{(i)}} \ll \lambda_{1} \mu, \quad \text { for } i=1,2,3 .
$$

We show the argument for $i=1$. In order to prove (3.17), consider an arbitrary horizontal line $\Gamma$ in $Q^{(i)}$ and let $\tilde{Q}$ be the larger of the two rectangles confined by $\Gamma$ and $\partial Q$, in particular $|\tilde{Q}| \sim 1$. We note that on both vertical boundaries of $\tilde{Q}$, we have (3.16) and hence $\left|\left\langle\partial_{1} u_{2}\right\rangle_{\tilde{Q}}\right| \ll \lambda_{1} \mu$. Arguing as in the previous step of the proof, it follows that $\left|\left\langle\partial_{2} u_{1}\right\rangle_{\tilde{Q}}\right| \ll \lambda_{1} \mu$. Since on one horizontal boundary of $\tilde{Q}$ we have $\left\|u_{1}\right\|_{L^{\infty}} \ll \lambda_{1} \mu$, this yields, $\left\langle u_{1}\right\rangle_{\Gamma} \ll \lambda_{1} \mu$. This concludes the proof of (3.17).

Step 5: Contradiction. In this step, we use the diagonal part of the elastic energy to derive a contradiction. Indeed, we shall show that

$$
\left\langle u_{1}\right\rangle_{Q^{(3)}}-\left\langle u_{1}\right\rangle_{Q^{(1)}} \gtrsim \lambda_{1} \mu
$$

contradicting (3.17). To see (3.18), we choose $x_{1}^{*} \in I_{1}^{(1)}$ such that $f_{I_{2}} u_{1}\left(x_{1}^{*}, \tilde{y}_{2}\right) d \tilde{y}_{2}=$ $\left\langle u_{1}\right\rangle_{Q^{(1)}}$. We then have

$$
u_{1}\left(\tilde{y}_{1}, \tilde{y}_{2}\right)=u_{1}\left(x_{1}^{*}, \tilde{y}_{2}\right)+\int_{x_{1}^{*}}^{\tilde{y}_{1}} \partial_{1} u_{1}\left(\tilde{x}_{1}, \tilde{y}_{2}\right) d \tilde{x}_{1}
$$


Averaging over $\left(\tilde{y}_{1}, \tilde{y}_{2}\right) \in Q_{3}$, then yields

$$
\begin{aligned}
& \left\langle u_{1}\left(\tilde{y}_{1}, \tilde{y}_{2}\right)\right\rangle_{Q^{(3)}}=\left\langle u_{1}\left(x_{1}^{*}, \tilde{y}_{2}\right)+\int_{x_{1}^{*}}^{\tilde{y}_{1}} \partial_{1} u_{1}\left(\tilde{x}_{1}, \tilde{y}_{2}\right) d \tilde{x}_{1}\right\rangle_{Q^{(3)}} \\
& =\left\langle u_{1}\right\rangle_{Q^{(1)}}+\left\langle\int_{x_{1}^{*}}^{\tilde{y}_{1}} \lambda_{1} \chi\left(\tilde{x}_{1}, \tilde{y}_{2}\right) d \tilde{x}_{1}\right\rangle_{Q^{(3)}}+\left\langle\int_{x_{1}^{*}}^{\tilde{y}_{1}} \partial_{1} u_{1}\left(\tilde{x}_{1}, \tilde{y}_{2}\right)-\lambda_{1} \chi\left(\tilde{x}_{1}, \tilde{y}_{2}\right) d \tilde{x}_{1}\right\rangle_{Q^{(3)}} \\
& \gtrsim\left\langle u_{1}\right\rangle_{Q^{(1)}}+\lambda_{1} \mu+\left\langle\int_{x_{1}^{*}}^{\tilde{y}_{1}} \partial_{1} u_{1}\left(\tilde{x}_{1}, \tilde{y}_{2}\right)-\chi\left(\tilde{x}_{1}, \tilde{y}_{2}\right) \lambda_{1} d \tilde{x}_{1}\right\rangle_{Q^{(3)}},
\end{aligned}
$$

where the averages are taken in the variables $\left(\tilde{y}_{1}, \tilde{y}_{2}\right)$ and where we have used $\int_{Q^{(2)}} \chi \geq \mu$ in the last estimate. The last term on the right hand side can be estimated by application of Young's inequality,

$$
\left\langle\int_{x_{1}^{*}}^{\tilde{y}_{1}} \partial_{1} u_{1}\left(\tilde{x}_{1}, \tilde{y}_{2}\right)-\chi\left(\tilde{x}_{1}, \tilde{y}_{2}\right) \lambda_{1} d \tilde{x}_{1}\right\rangle_{Q^{(3)}} \lesssim\left\|\partial_{1} u_{1}\left(\tilde{x}_{1}, \tilde{y}_{2}\right)-\lambda_{1} \chi\left(\tilde{x}_{1}, \tilde{x}_{2}\right)\right\|_{L^{2}\left(Q^{(3)}\right)}
$$

which in view of (3.4) and (3.19) implies (3.18).

The following result will be needed in the proof for higher space dimensions. Note that this result does not only apply for characteristic functions, but instead works for any $\bar{\chi}$ taking values in $[0,1]$ :

Lemma 3.3. Suppose that the sets $Q^{(i)} \subset \mathbb{R}^{2}, i=1,2,3$, are given as in the proof of proposition 3.2. Furthermore suppose that (3.10) holds. Then we have for any $\bar{\chi} \in B V\left(\bigcup_{i=1}^{3} Q^{(i)},[0,1]\right)$,

$$
\inf _{u \in H^{1}\left(\mathbb{R}^{2}, \mathbb{R}^{2}\right)}\|e(u)-\bar{\chi} F\|_{L^{2}\left(\bigcup_{i=1}^{3} Q^{(i)}\right)}^{2} \gtrsim\|F\|^{2}\|\bar{\chi}\|_{L^{1}\left(Q_{2}\right)}^{2} .
$$

Proof. By assumption, the conclusion of Step 3 in the proof of lemma 3.2 is satisfied. We then can argue as in Step 4 and 5 to get (3.20).

We now turn to the proof of proposition 3.1 for general $d \geq 2$. In this case, the surface energy on the boundaries $\partial Q^{(i)}$ in generally is no longer discrete and hence the argument leading to (3.8)-(3.9) cannot be used. The idea is to recover an estimate related to $(3.9)$ by averaging out $(d-1)$ dimensions and by using an induction argument.

Lemma 3.4. Proposition 3.1 holds for all $d \geq 2$.

Proof. By the rescaling $x \mapsto x / R$ and a rotation, we may assume that $R=1$ and that $F=\operatorname{diag}\left(\lambda_{1}, \ldots, \lambda_{d}\right)$ where, without loss of generality, $\lambda_{1} \geq \ldots \geq \lambda_{d}$ and $\lambda_{1} \geq\left|\lambda_{d}\right|$. Proceeding by induction, we show that proposition 3.1 holds in $d$ dimensions if it holds both in $(d-1)$-dimensions and 2 dimensions. The case $d=2$ has been shown in lemma 3.2. By induction hypothesis, we hence assume that the the proposition holds for all $d^{\prime}$ with $2 \leq d^{\prime} \leq d-1$. We will argue by contradiction and hence (setting $\mu:=\int_{B_{\alpha_{d}}} \chi$ ), we assume

$$
\|e(u)-\chi F\|_{L^{2}\left(B_{1}\right)} \ll \lambda_{1} \mu .
$$


Step 1: Notation and choice of $Q^{(i)}$. The geometry is a natural generalization of the one used in the proof of lemma 3.2: We choose sets $Q^{(i)}=$ $I_{1}^{(i)} \times I_{2} \times H \subset B_{3 \alpha_{d}}$, where $H=\prod_{j=3}^{d} I_{j}$ and where $I_{1}^{(i)}=\left[x_{1}^{(i-1)}, x_{1}^{(i)}\right]$ and $I_{j}=\left[x_{j}^{(0)}, x_{j}^{(1)}\right]$ with $x_{j}^{(i-1)}<x_{j}^{(i)}$ for $i=1,2,3$ and $j=1, \ldots, d$. Also let $I_{1}:=\bigcup_{i} I_{1}^{(i)}$. We choose the sets such that $B_{\alpha_{d}} \subset Q^{(2)}$, in particular $\int_{Q^{(2)}} \chi \geq \mu$. Furthermore, we may assume that the side lengths of $Q^{(i)}$ are of order 1, i.e. $\left|I_{1}^{(i)}\right| \sim 1,\left|I_{j}\right| \sim 1$. We also define $\Pi$ as the extension through $B_{1}$ of all boundaries of the sets $Q^{(i)}$ with normal $e_{1}$ or $e_{2}$, i.e.

$$
\Pi:=\bigcup_{i=0}^{3} B_{1} \cap\left(\left\{x_{1}^{(i)}\right\} \times \mathbb{R}^{d-1}\right) \cup \bigcup_{j=0}^{1} B_{1} \cap\left(\mathbb{R} \times\left\{x_{2}^{(j)}\right\} \times \mathbb{R}^{d-2}\right) .
$$

By Fubini's theorem and by adjusting the sets slightly, we may assume that there is no concentration of energy and no concentration of the minority phase on $\Pi$, i.e.

$$
\begin{aligned}
\|e(u)-\chi F\|_{L^{2}(\Pi)} & \lesssim\|e(u)-\chi F\|_{L^{2}\left(B_{1}\right)} \stackrel{(3.21)}{\ll} \lambda_{1} \mu, \\
\|\nabla \chi\|_{\Pi} & \lesssim\|\nabla \chi\|_{B_{1}} \stackrel{(3.1)}{\ll} 1, \\
\|\chi\|_{L^{1}(\Pi)} & \lesssim\|\chi\|_{L^{1}\left(B_{1}\right)} \stackrel{(3.1)}{\ll} 1 .
\end{aligned}
$$

Step 2: Predominantly majority phase on part of boundary. For $d=2$, we have used that the restriction of the measure $\|\nabla \chi\|$ on $1-\mathrm{d}$ sets is discrete to strengthen (3.6)-(3.7) to (3.8)-(3.9). This is not possible for $d \geq 3$. Instead, we claim that we have only a small fraction of the minority phase on all surfaces of $\partial Q^{(i)}$ with normal $e_{1}$ and $e_{2}$, in the sense of

$$
\left\|\lambda_{2} \chi\right\|_{L^{1}\left(\bigcup_{i=0}^{3}\left\{x_{1}^{(i)}\right\} \times I_{2} \times H\right)}+\left\|\lambda_{1} \chi\right\|_{L^{1}\left(\bigcup_{i=0}^{3} \bigcup_{j=0}^{1} I_{1}^{(i)} \times\left\{x_{2}^{(j)}\right\} \times H\right)} \ll \lambda_{1} \mu,
$$

which can be seen as stronger version of (3.24). We present the argument for one estimate in (3.25) (the other arguments being analogous), i.e. we show

$$
\left\|\lambda_{2} \chi\right\|_{L^{1}\left(\left\{x_{1}^{(0)}\right\} \times I_{2} \times H\right)} \ll \lambda_{1} \mu \text {. }
$$

In order to prove $(3.26)$, consider $\tilde{\Pi}_{1} \supset I_{2} \times H$ where $\tilde{\Pi}_{1}$ is the projection of the set $\left(x_{1}^{(0)} \times \mathbb{R}^{d-1}\right) \cap B_{1}$ on the last $(d-1)$ components. Note that if $\alpha_{d}$ is sufficiently small, then for some $\tilde{x} \in \mathbb{R}^{d-1}, \rho \sim 1$, we have

$$
I_{2} \times H \subset \tilde{B}_{\alpha_{d-1} \rho}(\tilde{x}) \subset \tilde{B}_{\rho}(\tilde{x}) \subset \tilde{\Pi}_{1}
$$

where $\tilde{B}_{\gamma}(\tilde{x}) \subset \mathbb{R}^{d-1}$ is the $(d-1)$-dimensional ball with center $\tilde{x}$ and radius $\gamma$. Furthermore by $(3.23)-(3.24)$, the restriction of $\chi$ on the set $\left\{x_{1}^{(0)}\right\} \times \tilde{\Pi}_{1}$ satisfies (3.1). Finally, the restriction of $F$ to directions in $\Pi_{1}^{(0)}$ has norm $\geq \lambda_{2}$. By the induction hypothesis, it hence follows that

$$
\lambda_{2} \int_{\left\{x_{1}^{(0)}\right\} \times I_{2} \times H} \chi \lesssim\|e(u)-\chi F\|_{L^{2}\left(\left\{x_{1}^{(0)}\right\} \times \tilde{\Pi}_{1}\right)} \stackrel{(3.22)}{\ll} \lambda_{1} \mu,
$$


concluding the proof of (3.26).

Step 3: Reduction to 2-d. In this step, we reduce the argument to the twodimensional case by averaging out $H$-dependence. For this, we define $\bar{u}=\left(\bar{u}_{1}, \bar{u}_{2}\right)$ : $I_{1} \times I_{2} \rightarrow \mathbb{R}^{2}$ and $\bar{\chi}: I_{1} \times I_{2} \rightarrow[0,1]$ by

$$
\bar{u}_{i}\left(x_{1}, x_{2}\right):=f_{H} u_{i}\left(x_{1}, x_{2}, x_{\perp}\right) d x_{\perp}, \quad \bar{\chi}\left(x_{1}, x_{2}\right):=f_{H} \chi\left(x_{1}, x_{2}, x_{\perp}\right) d x_{\perp},
$$

where $i=1,2$. Correspondingly, we define the $2-$ d strain matrix $\tilde{F}:=\operatorname{diag}\left(\lambda_{1}, \lambda_{2}\right)$. We note that by Jensen's inequality we have

$$
\int_{I_{1} \times I_{2}}\|e(\bar{u})-\bar{\chi} \tilde{F}\|^{2} \lesssim \int_{Q}\|e(u)-\chi F\|^{2} .
$$

We claim that (after normalization) $\bar{u}_{1}$ is small on all horizontal boundaries and $\bar{u}_{2}$ is small on all vertical boundaries of the sets $I_{1} \times I_{2}^{(i)}$, i.e.

$$
\left\|\bar{u}_{1}\right\|_{L^{\infty}\left(I_{1} \times\left\{x_{2}^{(j)}\right\}\right)}+\left\|\bar{u}_{2}\right\|_{L^{\infty}\left(\left\{x_{1}^{(i)}\right\} \times I_{2}\right)} \ll \lambda_{1} \mu,
$$

where $i=0,1,2,3$ and $j=0,1$. Assuming for a moment that (3.28) holds, we can apply lemma 3.3 to get

$$
\|e(u)-\chi F\|_{L^{2}(Q)} \stackrel{(3.27)}{\gtrsim}\|e(\bar{u})-\bar{\chi} \tilde{F}\|_{L^{2}\left(I_{1} \times I_{2}\right)} \gtrsim \lambda_{1} \mu,
$$

which contradicts (3.21). This concludes the proof of the lemma if (3.28) holds.

Step 4: Smallness of $\bar{u}$ on $\partial\left(I_{1} \times I_{2}^{(i)}\right)$. It remains to prove (3.28), which is done in this step. By a change of coordinates (as in Step 3 of the proof of Lemma 3.2 , we may assume

$$
\left\langle\bar{u}_{1}\right\rangle_{I_{1} \times\left\{x_{2}^{(j)}\right\}}=0 \quad \text { for } j=0,1 \quad \text { and } \quad\left\langle\bar{u}_{2}\right\rangle_{\left\{x_{1}^{(0)}\right\} \times I_{2}}=0 .
$$

It follows that for $j=0,1$, we have

$$
\begin{aligned}
& \left\|\bar{u}_{1}\right\|_{L^{\infty}\left(I_{1} \times\left\{x_{2}^{(j)}\right\}\right)} \stackrel{(3.29)}{\lesssim}\left\|\partial_{1} \bar{u}_{1}\right\|_{L^{1}\left(I_{1} \times\left\{x_{2}^{(j)}\right\}\right)} \\
& \leq\left\|\partial_{1} \bar{u}_{1}-\lambda_{1} \bar{\chi}\right\|_{L^{1}\left(I_{1} \times\left\{x_{2}^{(j)}\right\}\right)}+\left\|\lambda_{1} \bar{\chi}\right\|_{L^{1}\left(I_{1} \times\left\{x_{2}^{(j)}\right\}\right)} \\
& \stackrel{(3.27)}{\lesssim}\left\|\partial_{1} u_{1}-\lambda_{1} \chi\right\|_{L^{2}\left(I_{1} \times\left\{x_{2}^{(j)}\right\} \times H\right)}+\left\|\lambda_{1} \bar{\chi}\right\|_{L^{1}\left(I_{1} \times\left\{x_{2}^{(j)}\right\}\right)} \stackrel{(3.22),(3.25)}{\ll} \lambda_{1} \mu,
\end{aligned}
$$

where we have used Jensen's inequality in the third estimate. From (3.30), we get

$$
\left|\left\langle\partial_{2} \bar{u}_{1}\right\rangle_{Q^{(i)}}\right| \ll \lambda_{1} \mu, \quad \text { for } i=1,2,3 .
$$

As in (3.14), estimate (3.31) yields control of $u_{2}$, i.e. we get $\left|\left\langle\partial_{1} \bar{u}_{2}\right\rangle_{Q^{(i)}}\right| \ll \lambda_{1} \mu$ for $i=1,2,3$. By (3.29), it then follows successively that the average of $\bar{u}_{2}$ is small on all vertical boundaries of $Q^{(i)}$, i.e.

$$
\left|\left\langle\bar{u}_{2}\right\rangle_{\left\{x_{1}^{(i)}\right\} \times I_{2}}\right| \ll \lambda_{1} \mu, \quad \text { for } i=0,1,2,3 .
$$


Now, as before, using control of elastic energy (3.22), we obtain

$$
\begin{aligned}
& \left\|\bar{u}_{2}\right\|_{L^{\infty}\left(\left\{x_{1}^{(i)}\right\} \times I_{2}\right)} \lesssim\left|\left\langle\bar{u}_{2}\right\rangle_{\left\{x_{1}^{(i)}\right\} \times I_{2}}\right|+\left\|\partial_{2} \bar{u}_{2}-\bar{\chi} \lambda_{2}\right\|_{L^{1}\left(\left\{x_{1}^{(i)}\right\} \times I_{2}\right)}+\left\|\lambda_{2} \bar{\chi}\right\|_{L^{1}\left(\left\{x_{1}^{(i)}\right\} \times I_{2}\right)} \\
& \quad \lesssim\left|\left\langle\bar{u}_{2}\right\rangle_{\left\{x_{1}^{(i)}\right\} \times I_{2}}\right|+\left\|\partial_{2} u_{2}-\chi \lambda_{2}\right\|_{L^{2}\left(\left\{x_{1}^{(i)}\right\} \times I_{2} \times H\right)}+\left\|\lambda_{2} \bar{\chi}\right\|_{L^{1}\left(\left\{x_{1}^{(i)}\right\} \times I_{2}\right)} \\
& \quad \ll \lambda_{1} \mu, \quad \text { for } i=1,2,3,
\end{aligned}
$$

where we have used (3.32), (3.21) and (3.25) in the last estimate. This concludes the proof of (3.28) and hence of lemma 3.4.

We next address a lower bound for incompatible strains:

Proposition 3.5 (Lower bound for incompatible strains). We have

$$
\inf _{u \in H^{1}\left(\mathbb{R}^{d}, \mathbb{R}^{d}\right)}\|e(u)-\chi F\|_{L^{2}\left(\mathbb{R}^{d}\right)}^{2} \geq \inf _{P \in \mathcal{V}(d)}\|F-P\|^{2}\|\chi\|_{L^{1}\left(\mathbb{R}^{d}\right)} .
$$

Proof. By a rotation we may assume that $F=\operatorname{diag}\left(\lambda_{1}, \ldots, \lambda_{d}\right)$ and, without loss of generality, $\lambda_{1} \geq \ldots \geq \lambda_{d}$ and $\lambda_{1} \geq\left|\lambda_{d}\right|$. Furthermore, we may assume that $u$ is a minimiser for fixed $\chi$. Then, in view of lemma 4.1, the elastic energy can be expressed in Fourier variables as

$$
\inf _{u \in H^{1}\left(\mathbb{R}^{d}, \mathbb{R}^{d}\right)} \int_{\mathbb{R}^{d}}\|e(u)-\chi F\|^{2}=\int_{\mathbb{R}^{d}}|\hat{\chi}|^{2} \Phi(n) d \xi,
$$

where $n=\xi /|\xi|$ and where $\Phi(n)=\|F\|^{2}-2\|F n\|^{2}+\langle n, F n\rangle^{2}$. By Parseval's identity and since $\chi$ is a characteristic function, it follows that

$$
\int_{\mathbb{R}^{d}}\|e(u)-\chi F\|^{2} \geq \inf _{|n|=1} \Phi(n) \int_{\mathbb{R}^{d}}|\hat{\chi}|^{2}=\inf _{|n|=1} \Phi(n) \int_{\mathbb{R}^{d}}|\chi|^{2}=\inf _{|n|=1} \Phi(n) \int_{\mathbb{R}^{d}} \chi .
$$

The proof is concluded by the lower bound on $\Phi$ in lemma 4.2.

\section{(b) Proof of theorem 2.1}

In this section, we give the proof of theorem 2.1. We first note that by rescaling in length, one of the parameters $\mu, \eta,\|F\|$ can be scaled to 1 . We choose to remove $\eta$, i.e. we rescale $x=\eta^{-1} \hat{x}, F=\eta \hat{F}, \mu=\eta^{-d} \hat{\mu}$ and $E=\eta^{2-d} \hat{E}$. Furthermore, since the energy dependence on $\gamma$ is trivial, we may assume $\gamma=0$ in this section. Skipping the hats in the sequel, the non-dimensionalized version of the energy is then given by

$$
E(\chi, u)=\int_{\mathbb{R}^{d}}|\nabla \chi|+\int_{\mathbb{R}^{d}}\|e(u)-\chi F\|^{2}
$$

while theorem 2.1 is equivalent to

Theorem 3.6. For any $d \geq 2$, there is a constant $C$ (depending only on $d$ ) such that

$$
\inf _{(\chi, u) \in \mathcal{A}(\mu)} E(\chi, u) \leq \inf _{P \in \mathcal{V}(d)}\|F-P\|^{2} \mu+C\left\{\begin{array}{ll}
\mu^{\frac{d-1}{d}} & \text { if } \mu \leq\|F\|^{-2 d}, \\
\|F\|^{\frac{2 d-2}{2 d-1}} \mu^{\frac{2 d-2}{2 d-1}} & \text { if } \mu \geq\|F\|^{-2 d}
\end{array} .\right.
$$




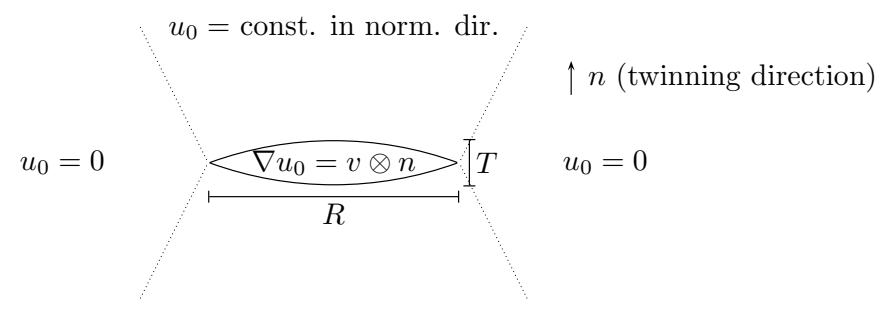

Figure 2. Construction of $u_{0}$ for inclusion with large volume

Also, there are constants $c_{1}, c_{2}$ (depending only on $d$ ) such that

$$
\inf _{(\chi, u) \in \mathcal{A}(\mu)} E(\chi, u) \geq \inf _{P \in \mathcal{V}(d)}\|F-P\|^{2} \mu+c_{1} \mu^{\frac{d-1}{d}}
$$

and

$$
\inf _{(\chi, u) \in \mathcal{A}(\mu)} E(\chi, u) \geq c_{2}\|F\|^{\frac{2 d-2}{2 d-1}} \mu^{\frac{2 d-2}{2 d-1}} .
$$

We shall discuss the upper and lower bounds separately.

Proof of theorem 3.6 - Upper bound. This result is well understood in the physical literature, see e.g. (Khachaturyan 1982). We give a simple proof which does not require the use of Fourier transform.

Part 1: The case of large inclusions. We first give the construction for the case of larger inclusions, i.e when $\mu \gg\|F\|^{-2 d}$. In this case, the shape is determined by a balance of elastic energy and surface energy. The idea is to choose the inclusion to have approximately the shape of a thin disc $Q_{T, R}$ with diameter $R$ and thickness $T$ where $T \ll R$. The disc is oriented such that the two large surfaces are aligned with one of the twin planes between $F$ and 0 . Since the desired result is a scaling law, it will be sufficient to specify how $T$ and $R$ scale with the parameters. In particular, we do not need to optimize the precise shape of the disc.

The construction is as follows: Let $P$ be the projection of $F$ on the set of compatible strains. In particular, $P$ has the representation $P=\frac{1}{2}(v \otimes n+n \otimes v)$ for some $v, n \in \mathbb{R}^{d}$ with $\|n\|=1$ and furthermore $\|v \otimes n\| \lesssim\|F\|$, see lemma 4.2. The construction is symmetric with respect to the cylindrical coordinates $z:=\langle x, n\rangle$, $r:=|x-\langle x, n\rangle n|$.

Consider two points $x^{(1)}=-x^{(2)}$ on the axis $r=0$ with distance $d$ and consider the intersection $B_{\rho}\left(x^{(1)}\right) \cap B_{\rho}\left(x^{(2)}\right)$, where $\rho>0$. Now for any $0<T \ll R$, we can adjust $d$ and $\rho$ such that the intersection is a lens with thickness of order $T$ and diameter of order $R$. We next define $\chi$ by

$$
\chi:=\chi_{Q_{T, R}}, \quad \text { where } \quad Q_{T, R}:=B_{\rho}\left(x^{(1)}\right) \cap B_{\rho}\left(x^{(2)}\right) .
$$

Assuming that the consistency condition $T \ll R$ holds, the interfacial energy for this configuration is estimated by $\int_{\mathbb{R}^{d}}|\nabla \chi| \lesssim R^{d-1}$. It remains to choose the deformation $u$. For this, we define $u_{0}: \mathbb{R}^{d} \rightarrow \mathbb{R}^{d}$ by $u_{0}(0)=0$ and $\nabla u_{0}=v \otimes n$ in $Q_{T, R}$. Outside of $Q_{T, R}$, we let $u_{0}$ be constant on all lines which are normal to the surface $\partial Q_{T, R}$. In the remaining area, we set $u_{0}=0$, see figure 2 . In particular,

$$
\left\|\nabla u_{0}(x)\right\| \lesssim\|F\|\left\{\begin{array}{ll}
1 & \text { for } x \in Q_{T, R}, \\
T R^{-1} & \text { for } x \notin Q_{T, R},
\end{array} \quad\left|u_{0}(x)\right| \lesssim\|F\| T \quad \text { for } x \in \mathbb{R}^{d} .\right.
$$


Let $0 \leq \zeta \leq 1$ be a smooth cut-off function such that $\zeta=1$ in $B_{R}$ and $\zeta=0$ outside $B_{2 R}$. We choose $\zeta$ such that furthermore $|\nabla \zeta| \lesssim 1 / R$. We then define $u: \mathbb{R}^{d} \rightarrow \mathbb{R}^{d}$ by $u:=\zeta u_{0}$. Correspondingly, the elastic energy is estimated as follows

$$
\int_{\mathbb{R}^{d}}\|e(u)-\chi F\|^{2} \leq\|F-P\|^{2}\left|Q_{T, R}\right|+C \int_{\mathbb{R}^{d} \backslash Q_{T, R}}\|\nabla u\|^{2},
$$

where

$$
\int_{\mathbb{R}^{d} \backslash Q_{T, R}}\|\nabla u\|^{2} \lesssim \int_{B_{2 R} \backslash B_{R}}|\nabla \zeta|^{2}\left|u_{0}\right|^{2}+\int_{B_{2 R} \backslash B_{R}}|\zeta|^{2}\left\|\nabla u_{0}\right\|^{2} \stackrel{(3.36)}{\lesssim}\|F\|^{2} R^{d-2} T^{2} .
$$

We choose $T$ such that the volume constraint (2.3) is satisfied, i.e. $\left|Q_{T, R}\right|=\mu$ and in particular, $R^{d-1} T \sim \mu$. It follows that

$$
E(\chi, u)-\|F-P\|^{2} \mu \lesssim R^{d-1}+\|F\|^{2} \mu^{2} R^{-d} \lesssim\|F\|^{\frac{2 d-2}{2 d-1}} \mu^{\frac{2 d-2}{2 d-1}},
$$

where we have chosen the optimal radius $R=\|F\|^{\frac{2}{2 d-1}} \mu^{\frac{2}{2 d-1}}$. One can check that $R, T$, defined above, satisfy the consistency condition $T \ll R$ if $\mu \gg\|F\|^{-2 d}$ is satisfied. This concludes the proof of the upper bound for large inclusions.

Part 2: The case of small inclusions. It remains to consider the case $\mu \lesssim\|F\|^{-2 d}$. In this case the surface energy dominates. Accordingly, we choose the inclusion to have the shape of a ball with volume $\mu$ and radius of size $R \sim \mu^{1 / d}$, i.e. $\chi:=\chi_{B_{R}}$. In particular, the interfacial contribution of the energy is estimated by

$$
\int_{\mathbb{R}^{d}}|\nabla \chi| \lesssim R^{d-1} \lesssim \mu^{\frac{d-1}{d}}
$$

Now, let $\zeta$ be a smooth cut-off function with $0 \leq \zeta \leq 1$ and such that $\zeta=1$ in $B_{R}$ and $\zeta=0$ outside $B_{2 R}$. We may furthermore assume that $|\nabla \zeta| \lesssim 1 / R$. We define $u_{0}: \mathbb{R}^{d} \rightarrow \mathbb{R}^{d}$ by $u_{0}(0)=0$ and $\nabla u_{0}=P$. In particular,

$$
\left|u_{0}(x)\right| \lesssim R\|F\|, \quad\left\|\nabla u_{0}(x)\right\| \lesssim\|F\|, \quad \text { for all } x \in B_{2 R} .
$$

Now, choosing $u:=\zeta u_{0}$, the elastic energy can be estimated as follows

$$
\begin{aligned}
\int_{\mathbb{R}^{d}}\|e(u)-F\|^{2} & \leq\|F-P\|^{2}\left|B_{R}\right|+C \int_{B_{2 R}}\left(\left|u_{0}\right|^{2}|\nabla \zeta|^{2}+C\left\|\nabla u_{0}\right\|^{2}|\zeta|^{2}\right) \\
& \leq\|F-P\|^{2}\left|B_{R}\right|+C\|F\|^{2} R^{d} \leq\|F-P\|^{2}\left|B_{R}\right|+C R^{d-1} \\
& \leq\|F-P\|^{2} \mu+C \mu^{\frac{d-1}{d}}
\end{aligned}
$$

where we have used $\mu \leq\|F\|^{-2 d}$ in the third inequality. Estimates (3.37) and (3.38) together yield the upper bound for small inclusions.

Proof of theorem 3.6 - Lower bounds. The first lower bound (3.34) is easy. Proposition 3.5 gives a lower bound for the elastic term, and the isoperimetric inequality gives a lower bound for the surface energy term. Combining the two results gives (3.34).

The only remaining task is to prove

$$
\inf _{(\chi, u) \in \mathcal{A}(\mu)} E(\chi, u) \gtrsim\|F\|^{\frac{2 d-2}{2 d-1}} \mu^{\frac{2 d-2}{2 d-1}} .
$$


In order to show (3.39), we combine an application of proposition 3.1 with a decomposition argument. Let $M:=\operatorname{supp} \chi$. Without loss of generality we assume that all $x \in M$ are points of density 1 of $M$. For all $x \in M$, let

$$
R(x):=\inf \left\{r: r^{-d}\left|M \cap B_{r}(x)\right| \leq c_{0} \min \left\{1,\left|M \cap B_{r}(x)\right|^{\frac{-1}{2 d-1}}\|F\|^{\frac{-2 d}{2 d-1}}\right\}\right\},
$$

where $c_{0}$ is a sufficiently small universal constant to be fixed later. We note that both sides of the inequality in (3.40) are continuous functions in $r$. Furthermore, the inequality in (3.40) is not satisfied for $r=0$ (since $x$ has density 1 in $M$ ), but always satisfied in the limit $r \rightarrow \infty$ (since $|M|<\infty$ ). This ensures the existence of $R(x)$ satisfying (3.40). Furthermore, we observe that $R=R(x)$ satisfies one of the following conditions: Either

$$
\left|M \cap B_{R}(x)\right| \leq\|F\|^{-2 d} \quad \text { and } \quad\left|M \cap B_{R}(x)\right|=c_{0} R^{d},
$$

or

$$
\left|M \cap B_{R}(x)\right|>\|F\|^{-2 d} \quad \text { and } \quad\left|M \cap B_{R}(x)\right|^{\frac{2 d}{2 d-1}}=c_{0}\|F\|^{\frac{-2 d}{2 d-1}} R^{d}
$$

Let us explain how definition (3.40) is motivated by the two constructions in the proof of the upper bound: In fact, for each of these constructions, take the smallest ball that covers $\operatorname{supp} \chi$ and consider the density of the minority phase in this ball. Then, up to the constant $c_{0}$, in (3.41) the density of the minority phase in $B_{R}(x)$ corresponds to the density of the upper bound construction for the case of small inclusions. Similarly, up to the constant $c_{0}$, the density of the minority phase in (3.42) corresponds to the density of the upper bound construction for the case of large inclusions.

Trivially, $M$ is covered by $\bigcup_{x \in M} B_{R(x) / 5}(x)$ and by (3.41)-(3.42), the radii $R(x)$ are uniformly bounded in terms of $\|F\|$ and $\mu$. Hence, by Vitali's covering lemma, there is an at most countable subset of points $x_{i} \in \mathbb{R}^{d}$ such that the balls $B_{R_{i} / 5}\left(x_{i}\right)$ are disjoint while $M$ is still covered by the balls $B_{R_{i}}\left(x_{i}\right)$. Let $R_{i}:=R\left(x_{i}\right), R_{i}^{\prime}:=$ $R_{i} / 5, R_{i}^{\prime \prime}:=\alpha_{d} R_{i} / 5$ (where $\alpha_{d}$ is the constant from Proposition 3.1) and let $B_{i}:=$ $B_{R_{i}}\left(x_{i}\right), B_{i}^{\prime}:=B_{R_{i}^{\prime}}\left(x_{i}\right), B_{i}^{\prime \prime}:=B_{R_{i}^{\prime \prime}}\left(x_{i}\right)$. In particular, $B_{i}^{\prime} \cap B_{j}^{\prime}=\emptyset$ for $i \neq j$ and $M \subseteq \bigcup_{i=1}^{\infty} B_{i}$. It follows that

$$
\sum_{i=1}^{\infty}\left|M \cap B_{i}\right| \geq \mu \quad \text { and } \quad E(u, \chi) \geq \sum_{i=1}^{\infty} E_{\mid B_{i}^{\prime}}(\chi, u)
$$

where $E_{\mid A}, A \subseteq \mathbb{R}^{d}$, is the fraction of the energy localized on the set $A$, i.e.

$$
E_{\mid A}(\chi, u):=\int_{A}|\nabla \chi|+\int_{A}\|e(u)-\chi F\|^{2} .
$$

We claim that the following two estimates hold

$$
\begin{aligned}
& \left|M \cap B_{i}^{\prime \prime}\right| \gtrsim\left|M \cap B_{i}^{\prime}\right| \gtrsim\left|M \cap B_{i}\right|, \\
& \left.E_{\mid B_{i}^{\prime}}(\chi, u) \gtrsim|| F\right|^{\frac{2 d-2}{2 d-1}}\left|M \cap B_{i}^{\prime \prime}\right|^{\frac{2 d-2}{2 d-1}} .
\end{aligned}
$$


The desired lower bound is then a consequence of (3.44)-(3.45). Indeed, we have

$$
\begin{aligned}
E(\chi, u) & \stackrel{(3.43)}{\geq} \sum_{i=1}^{\infty} E_{\mid B_{i}^{\prime}}(\chi, u) \stackrel{(3.45)}{\gtrsim}\|F\|^{\frac{2 d-2}{2 d-1}} \sum_{i=1}^{\infty}\left|M \cap B_{i}^{\prime \prime}\right|^{\frac{2 d-2}{2 d-1}} \\
& \stackrel{(3.44)}{\gtrless}\|F\|^{\frac{2 d-2}{2 d-1}} \sum_{i=1}^{\infty}\left|M \cap B_{i}\right|^{\frac{2 d-2}{2 d-1}} \stackrel{(3.43)}{\geq}\|F\|^{\frac{2 d-2}{2 d-1}} \mu^{\frac{2 d-2}{2 d-1}}
\end{aligned}
$$

where in the last estimate we have used that $\sum_{i} c_{i}^{\beta} \leq\left(\sum_{i} c_{i}\right)^{\beta}$ whenever $c_{i} \geq 0$ and $0 \leq \beta<1$. It remains to prove (3.44)-(3.45). In order to show (3.44), we note that by the minimality of $R$, the following estimates hold:

If $\left|M \cap B_{i}\right| \leq\|F\|^{-2 d}$ and $\left|M \cap B_{i}^{\prime \prime}\right| \leq\|F\|^{-2 d}$, then

$$
\frac{\left|M \cap B_{i}^{\prime \prime}\right|}{\left(\alpha_{d} R_{i} / 5\right)^{d}} \stackrel{(3.41)}{\geq} \frac{\left|M \cap B_{i}\right|}{R_{i}^{d}}
$$

Similarly, if $\left|M \cap B_{i}\right| \geq\|F\|^{-2 d}$ and $\left|M \cap B_{i}^{\prime \prime}\right| \geq\|F\|^{-2 d}$, then

$$
\frac{\left(\left|M \cap B_{i}^{\prime \prime}\right|\right)^{\frac{2 d}{2 d-1}}\|F\|^{\frac{2 d}{2 d-1}}}{\left(\alpha_{d} R_{i} / 5\right)^{d}} \stackrel{(3.42)}{\geq} \frac{\left|M \cap B_{i}\right|^{\frac{2 d}{2 d-1}}\|F\|^{\frac{2 d}{2 d-1}}}{R_{i}^{d}} .
$$

Finally, if $\left|M \cap B_{i}^{\prime \prime}\right| \leq\|F\|^{-2 d} \leq\left|M \cap B_{i}\right|$, then

$$
\frac{\left|M \cap B_{i}^{\prime \prime}\right|}{\left(\alpha_{d} R_{i} / 5\right)^{d}} \geq \frac{\left|M \cap B_{i}\right|^{\frac{2 d}{2 d-1}}\|F\|^{\frac{2 d}{2 d-1}}}{R_{i}^{d}} \geq \frac{\left|M \cap B_{i}\right|}{R_{i}^{d}} .
$$

Estimates (3.46)-(3.47) together yield (3.44).

In order to prove (3.45), we differentiate between three cases: In the first case, we assume that (3.41) holds. Since the density of the minority phase is much smaller than 1 in $B_{i}^{\prime \prime}$ and hence also in $B_{i}^{\prime}$, we get by the isoperimetric inequality that

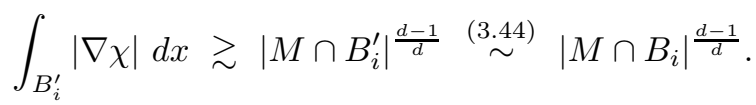

Note that in the above formula, the surface energy on $\partial B_{i}^{\prime}$ is not counted. This version of the isoperimetric inequality applies since we are in a low volume fraction case. It follows that

$$
E_{\mid B_{i}^{\prime}}(\chi, u) \geq \int_{B_{i}^{\prime}}|\nabla \chi| d x \stackrel{(3.48)}{\gtrsim}\left|M \cap B_{i}\right|^{\frac{d-1}{d}} \stackrel{(3.41)}{\gtrsim}\|F\|^{\frac{2 d-2}{2 d-1}}\left|M \cap B_{i}\right|^{\frac{2 d-2}{2 d-1}}
$$

In the second case, we assume that (3.42) holds and furthermore

$$
\int_{B_{i}^{\prime}}|\nabla \chi| d x \gtrsim R_{i}^{d-1}
$$

In view of $R_{i} \sim\|F\|^{2 /(2 d-1)}\left|M \cap B_{i}\right|^{2 /(2 d-1)}$, we immediately obtain

$$
E_{\mid B_{i}^{\prime}}(\chi, u) \geq \int_{B_{i}^{\prime}}|\nabla \chi| d x \stackrel{(3.49)}{\gtrsim} R_{i}^{d-1} \stackrel{(3.42)}{\sim}\|F\|^{\frac{2 d-2}{2 d-1}}\left|M \cap B_{i}\right|^{\frac{2 d-2}{2 d-1}} .
$$


It remains to consider the case when (3.42) holds and furthermore $\int_{B_{i}^{\prime}}|\nabla \chi| d x \ll$ $R_{i}^{d-1}$. In this case, choosing $c_{0}$ small enough, the assumptions of proposition 3.1 are satisfied (on $B_{i}^{\prime}$ ). An application of this proposition then yields

$$
E_{\mid B_{i}^{\prime}}(\chi, u) \gtrsim \frac{\left|M \cap B_{i}^{\prime \prime}\right|^{2}\|F\|^{2}}{R^{\prime d}} \stackrel{(3.44)}{\sim} \frac{\left|M \cap B_{i}\right|^{2}\|F\|^{2}}{R^{d}} \stackrel{(3.42)}{\sim}\|F\|^{\frac{2 d-2}{2 d-1}}\left|M \cap B_{i}\right|^{\frac{2 d-2}{2 d-1}} .
$$

The above estimates yield (3.45) which concludes the proof of (3.39) and hence of the theorem.

As we noted in the Introduction, it is natural to conjecture that the upper bound (2.4) is within a constant of being optimal. In the context of Theorem 3.6 this amounts to the conjecture that

$$
\inf _{(\chi, u) \in \mathcal{A}(\mu)} E(\chi, u) \geq \inf _{P \in \mathcal{V}(d)}\|F-P\|^{2} \mu+c \begin{cases}\mu^{\frac{d-1}{d}} & \text { if } \mu \leq\|F\|^{-2 d}, \\ \|F\|^{\frac{2 d-2}{2 d-1}} \mu^{\frac{2 d-2}{2 d-1}} & \text { if } \mu \geq\|F\|^{-2 d}\end{cases}
$$

Our methods seem incapable of giving such a conclusion, since the (real-space) argument used to prove (3.34) is insensitive to the value of $\inf _{P \in \mathcal{V}(d)}\|F-P\|^{2}$, while the (Fourier and Sobolev-estimate-based) argument used to prove (3.35) treats the elastic and perimeter terms separately. To do better, it would seem necessary to find a Fourier-based argument that treats the elastic and perimeter terms together.

\section{Two diffuse-interface models}

Diffuse-interface models are widely used in the literature on elastic phase transformations; recent examples include the work by Poduri \& Chen (1996) and Zhang et al. $(2007,2008)$. We present two diffuse-interface variants of our model $(2.2)$ and show that in the absence of bulk energy, i.e. when $\gamma=0$, the scaling of the minimal energy for the diffuse-interface models is the same as the one of the sharp-interface model.

In the first model, the energy is formulated in terms of the strain $e(u)$. We set

$$
\tilde{\mathcal{E}}_{1}(u)=\frac{\eta^{2}}{\|F\|^{4}} \int_{\mathbb{R}^{d}}\|\nabla e(u)\|^{2}+\int_{\mathbb{R}^{d}} \mathcal{W}_{1}(e(u))
$$

with double-well potential given by $\mathcal{W}_{1}(e):=\min \left\{\|e\|^{2},\|e-F\|^{2}\right\}$ where $F$ is (as usual) a symmetric matrix. The second model we want to discuss is given by

$$
\tilde{\mathcal{E}}_{2}(\tilde{\chi}, u)=\frac{\eta^{2}}{\|F\|^{2}} \int_{\mathbb{R}^{d}}|\nabla \tilde{\chi}|^{2}+\|F\|^{2} \int_{\mathbb{R}^{d}} W_{1}(\tilde{\chi})+\int_{\mathbb{R}^{d}}\|e(u)-\tilde{\chi} F\|^{2},
$$

where the standard double well potential $W_{1}(t):=t^{2}(1-t)^{2}$ penalizes the deviation of the order parameter $\tilde{\chi} \in H^{1}\left(\mathbb{R}^{d}\right)$ from a characteristic function. Model (4.1) is a strain-gradient version of (2.2). Models like (4.2) are often preferred for numerical work since the minimization over $u$ (given $\tilde{\chi}$ ) can be efficiently computed using FFT (Zhang et al. 2008). 
In (4.2) the "phase" is determined by a scalar-valued order parameter $\tilde{\chi}$. In connection with (4.1) it is convenient to define an analogous scalar-valued order parameter by

$$
\tilde{\chi}(x):=(2\|F\|)^{-1}(\|e(u)\|-\|e(u)-F\|+\|F\|) .
$$

Note that $0 \leq \tilde{\chi} \leq 1$ by the triangle inequality, and

$$
|\nabla \tilde{\chi}| \lesssim\|F\|^{-1}\|\nabla e(u)\| \text {. }
$$

As in the sharp-interface setting, we want to characterize the energy of inclusions with fixed volume of the minority phase. For this, we choose

$$
\tilde{\mu}(\tilde{\chi}):=|\{\tilde{\chi}>1 / 2\}|
$$

for both models (4.1)-(4.2); accordingly, the admissible functions for $\tilde{\mathcal{E}}_{1}$ and $\tilde{\mathcal{E}}_{2}$ are

$$
\begin{gathered}
\tilde{\mathcal{A}}_{1}(\mu):=\left\{u \in H^{1}\left(\mathbb{R}^{d}, \mathbb{R}^{d}\right): \tilde{\mu}(\tilde{\chi})=\mu\right\}, \\
\tilde{\mathcal{A}}_{2}(\mu):=\left\{(\tilde{\chi}, u) \in H^{1}\left(\mathbb{R}^{d}\right) \times H^{1}\left(\mathbb{R}^{d}, \mathbb{R}^{d}\right): \tilde{\mu}(\tilde{\chi})=\mu\right\} .
\end{gathered}
$$

Another possibility would be to use the $L^{1}$-norm of $\tilde{\chi}$ in definition (4.5). However, this is not a good notion for our purpose since then minimisers for fixed volume would tend to spread out to infinity (never approaching a phase transformation).

Neglecting bulk energy (i.e. setting $\gamma=0$ ), theorem 2.1 can be generalized to the above diffuse-interface models as stated in theorem 2.3. The proof proceeds as follows:

Proof of theorem 2.3. By the same rescaling as in $\S 3 \mathrm{~b}$, we may assume $\eta=1$. The proof of the upper bound for the two diffuse-interface energies is easy and follows by replacing the sharp interfaces in the constructions in the proof of theorem 3.6 by diffuse-interfaces with thickness of order $\eta /\|F\|^{2}$. Therefore the only nontrivial task is to prove lower bounds for $\tilde{\mathcal{E}}_{1}$ and $\tilde{\mathcal{E}}_{2}$.

We focus first on $\tilde{\mathcal{E}}_{2}$. Given $(\tilde{\chi}, u) \in \tilde{\mathcal{A}}_{2}(\tilde{\mu})$ we begin by constructing an appropriate sharp-interface function $\chi$. For this, we observe that

$$
\frac{1}{\|F\|^{2}} \int_{\mathbb{R}^{d}}|\nabla \tilde{\chi}|^{2}+\|F\|^{2} \int_{\mathbb{R}^{d}} W_{1}(\tilde{\chi}) \gtrsim \int_{\mathbb{R}^{d}}|\nabla \tilde{\chi}| \sqrt{W_{1}(\tilde{\chi})} \gtrsim \int_{1 / 4 \leq \tilde{\chi} \leq 1 / 2}|\nabla \tilde{\chi}| .
$$

It follows by the co-area formula and Fubini that there is $c^{*} \in(1 / 4,1 / 2)$ such that

$$
\frac{1}{\|F\|^{2}} \int_{\mathbb{R}^{d}}|\nabla \tilde{\chi}|^{2}+\|F\|^{2} \int_{\mathbb{R}^{d}} W_{1}(\tilde{\chi}) \gtrsim \int_{\mathbb{R}^{d}}|\nabla \chi|
$$

where $\chi \in B V\left(\mathbb{R}^{d},\{0,1\}\right)$ is defined by $\chi(x)=0$, if $\tilde{\chi}(x) \leq c^{*}$ and $\chi(x)=1$ if $\tilde{\chi}(x)>c^{*}$. In particular, since $c^{*}<1 / 2$ and in view of (4.5) we have $\|\chi\|_{L^{1}} \gtrsim \tilde{\mu}(\tilde{\chi})$. It remains to give a lower bound for the elastic energy. We have

$$
\tilde{E}_{\mathrm{el}}:=\int_{\mathbb{R}^{d}}\|e(u)-\tilde{\chi} F\|^{2}=\int_{\mathbb{R}^{d}}\|e(u)-\chi F\|^{2}-R,
$$


where one easily calculates that

$$
\begin{aligned}
R & =\int_{\mathbb{R}^{d}}(2(e(u)-\tilde{\chi} F)+(\tilde{\chi}-\chi) F):(\tilde{\chi}-\chi) F \lesssim \tilde{E}_{\mathrm{el}}+\int_{\mathbb{R}^{d}}\|F\|^{2}|\chi-\tilde{\chi}|^{2} \\
& \lesssim \tilde{E}_{\mathrm{el}}+\|F\|^{2} \int_{\mathbb{R}^{d}} W_{1}(\tilde{\chi}) \lesssim \tilde{\mathcal{E}}_{2}(\tilde{\chi}, u) .
\end{aligned}
$$

By (4.6), (4.7) and (4.8), it follows that $\tilde{\mathcal{E}}_{2}(\tilde{\chi}, u) \gtrsim E(\chi, u)-\tilde{\mathcal{E}}_{2}(\tilde{\chi}, u)$, whence $\tilde{\mathcal{E}}_{2}(\tilde{\chi}, u) \gtrsim E(\chi, u)$. Since $\|\chi\|_{L^{1}} \gtrsim \tilde{\mu}(\tilde{\chi})$ and since the scaling law (2.7) for the minimal energy is monotone in $\mu$ when $\gamma=0$, this means that

$$
\inf _{u \in \tilde{\mathcal{A}}_{2}(\mu)} \tilde{\mathcal{E}}_{2}(u) \gtrsim \inf _{(\chi, u) \in \mathcal{A}(\mu)} \mathcal{E}(\chi, u) .
$$

Our final task is to bound $\tilde{\mathcal{E}}_{1}$ from below by $\tilde{\mathcal{E}}_{2}$. Given $u \in \tilde{\mathcal{A}}_{1}(\tilde{\mu})$, we define $\tilde{\chi}$ by (4.3), and we note that by definition $(\tilde{\chi}, u) \in \tilde{\mathcal{A}}_{2}(\mu)$. It is clear from (4.4) that

$$
\frac{\eta^{2}}{\|F\|^{2}} \lesssim \frac{\eta^{2}}{\|F\|^{4}}\|\nabla e(u)\|^{2}
$$

It is also easy to see that

$$
\|F\|^{2} \tilde{\chi}^{2}(1-\tilde{\chi})^{2}+\|e(u)-\tilde{\chi} F\|^{2} \lesssim \min \left\{\|e\|^{2},\|e-F\|^{2}\right\}
$$

(the proof uses the fact that $\tilde{\chi}(e)$ is a Lipschitz-continuous function of $e$ with Lipschitz constant of order $\|F\|^{-1}$, and the properties that $\tilde{\chi}(0)=0$ and $\tilde{\chi}(F)=1$ ). Thus $\tilde{\mathcal{E}}_{1}(u) \gtrsim \tilde{\mathcal{E}}_{2}(\tilde{\chi}, u)$ which concludes the proof.

ACKNOWLEDGEMENT:The authors thank the anonymous referees for thoughtful comments which substantially improved the paper. R. Kohn furthermore gratefully acknowledges support from NSF grant DMS-0807347.

\section{Appendix A.}

The Fourier representation of the elastic field has been extensively studied e.g. by Khachaturyan (1982). For the convenience of the reader, we give the derivation following Capella \& Otto (2009):

Lemma 4.1. For any $\chi \in L^{2}\left(\mathbb{R}^{d}\right)$, we have (setting $n:=\xi /|\xi|$ )

$$
\inf _{u \in H^{1}\left(\mathbb{R}^{d}, \mathbb{R}^{d}\right)} \int_{\mathbb{R}^{d}}\|e(u)-\chi F\|^{2}=\int_{\mathbb{R}^{d}}|\hat{\chi}|^{2} \Phi(n) d \xi .
$$

where $\Phi(n)=\|F\|^{2}-2|F n|^{2}+\langle n, F n\rangle^{2}$.

Proof. Fourier transformation of the elastic energy yields

$$
\int_{\mathbb{R}^{d}}\|e(u)-\chi F\|^{2} d x=\int_{\mathbb{R}^{d}}\left\|\frac{\mathrm{i}}{2}(\xi \otimes \hat{u}+\hat{u} \otimes \xi)-\hat{\chi} F\right\|^{2} d \xi .
$$

Taking the first variation in (A 2), we get that for all $\xi \in \mathbb{R}^{d}, \zeta \in \mathbb{C}^{d}$

$$
\operatorname{Re}(\xi \otimes \zeta+\zeta \otimes \xi):(\xi \otimes \hat{u}+\hat{u} \otimes \xi+2 \mathrm{i} \hat{\chi} F)=0,
$$


where we used $\hat{u}(-\xi)=\overline{\hat{u}(\xi)}, \mathrm{i} \hat{\chi}(-\xi)=\mathrm{i} \overline{\hat{\chi}(\xi)}$. Since $F$ is symmetric, it follows that

$$
\langle\xi, \hat{u}\rangle \xi+|\xi|^{2} \hat{u}+2 \mathrm{i} \chi F \xi=0 .
$$

Multiplying this equation with $\xi$, one gets $\hat{u}(\xi)=\mathrm{i} \hat{\chi}|\xi|^{-1}(\langle n, F n\rangle n-2 F n)$ with $n:=\xi /|\xi|$. Inserting this formula into (A 2), a straightforward calculation then yields (A 1).

The following lemma characterises the set of compatible strains $\mathcal{V}(d)$ :

Lemma 4.2. Suppose that $F \in \mathcal{S}(d)$ has eigenvalues $\lambda_{1} \geq \ldots \geq \lambda_{d}$ (counted by multiplicity) with $\lambda_{1} \geq\left|\lambda_{d}\right|$. Then

$$
\inf _{P \in \mathcal{V}(d)}\|F-P\|^{2}=\inf _{|n|=1} \Phi(n)=\lambda_{2}^{2}+\ldots+\lambda_{d-1}^{2}+\max \left\{\lambda_{d}, 0\right\}^{2},
$$

where $\Phi(n)=\|F\|^{2}-2|F n|^{2}+\langle n, F n\rangle^{2}$. In particular,

$$
F \in \mathcal{V}(d) \Leftrightarrow \lambda_{1} \lambda_{d} \leq 0 \text { and } \lambda_{j}=0 \text { for } 2 \leq j \leq d-1 \text {. }
$$

Proof of lemma 4.2. To show the first equality in (A 3), we write $P=(u \otimes v+v \otimes$ $u) / 2$. The equality then follows by using the Euler-Lagrange equation as in the proof of lemma 4.1 (with $\hat{u}$ replaced by $u$ and $\xi$ replaced by $v$ ). To show the second equality in (A 3) it suffices to prove that

$$
\inf _{P \in \mathcal{V}(d)}\|F-P\|^{2} \leq \lambda_{2}^{2}+\ldots+\lambda_{d-1}^{2}+\max \left\{\lambda_{d}, 0\right\}^{2} \leq \inf _{|n|=1} \Phi(n),
$$

since we just have shown that the extreme right and left terms in (A 4) are equal. Let $e_{1}$ and $e_{d}$ be the eigenvectors for $\lambda_{1}$ and $\lambda_{d}$ and set

$$
u=\lambda_{1}^{1 / 2} e_{1}+\left(-\min \left\{\lambda_{d}, 0\right\}\right)^{1 / 2} e_{d} \quad \text { and } \quad v=\lambda_{1}^{1 / 2} e_{1}-\left(-\min \left\{\lambda_{d}, 0\right\}\right)^{1 / 2} e_{d}
$$

The choice $P=(u \otimes v+v \otimes u) / 2$ then leads to $P=\lambda_{1}\left(e_{1} \otimes e_{1}\right)+\min \left\{\lambda_{d}, 0\right\}\left(e_{d} \otimes e_{d}\right)$, which yields the left inequality in (A 4$)$. We turn to the right inequality: Note that, substituting $n_{1}^{2}=1-n_{2}^{2}-\ldots-n_{d}^{2}$, a straightforward calculation yields

$$
\begin{aligned}
\Phi(n) & =\sum_{j=2}^{d}\left(\lambda_{j}+\left(\lambda_{1}-\lambda_{j}\right) n_{j}^{2}\right)^{2}+\sum_{2 \leq i<j \leq d} 2\left(\lambda_{1}-\lambda_{i}\right)\left(\lambda_{1}-\lambda_{j}\right) n_{i}^{2} n_{j}^{2} \\
& =: \sum_{j=2}^{d} \Phi_{j}\left(n_{j}\right)+\sum_{2 \leq i<j \leq d} \Phi_{i j}\left(n_{i}, n_{j}\right) .
\end{aligned}
$$

where $\Phi_{j}\left(n_{j}\right) \geq 0$ and $\Phi_{i j}\left(n_{i}, n_{j}\right) \geq 0$. If $\lambda_{j} \geq 0$, then $\Phi_{j}\left(n_{j}\right) \geq \lambda_{j}^{2}$. This already yields (A 4) if $\lambda_{d} \geq 0$. Otherwise there is $2 \leq N \leq d$ such that $\lambda_{N-1} \geq 0$ and $\lambda_{N}<0$. Let $\alpha_{j}:=-\lambda_{j}$, hence $0<\alpha_{N} \leq \ldots \leq \alpha_{d}$, and $x_{j}:=\left(\lambda_{1}-\lambda_{j}\right) n_{j}^{2}$. A short calculation shows that, to conclude the proof of (A 4$)$, it is enough to show

$$
\min _{x_{N}, \ldots, x_{d} \geq 0} \varphi\left(x_{N}, \ldots, x_{d}\right) \geq-\alpha_{d}^{2}
$$


where

$$
\varphi\left(x_{N}, \ldots, x_{d}\right)=\sum_{j=N}^{d} x_{j}^{2}-2 \sum_{j=N}^{d} \alpha_{j} x_{j}+2 \sum_{N \leq i<j \leq d} x_{i} x_{j},
$$

The result now follows by straightforward minimization which is sketched below: Clearly, in order to prove (A 5), we may assume that $\alpha_{i} \neq \alpha_{j}$ for $i \neq j$ (the general case follows by continuity of (A 6) in the coefficients $\alpha_{j}$ ). Derivating (A 6$)$ in $x_{j}$ yields $\partial_{j} \varphi=x_{N}+\ldots+x_{d}-\alpha_{j}$ for $j=N, \ldots, d$ so that at every point, the partial derivative can only vanish in a single direction (since $\alpha_{i} \neq \alpha_{j}$ for $i \neq j$ ). It follows that the minimum is not achieved in the interior, but instead at a point where all $x_{j}=0$ except at most one $x_{i} \neq 0$. A short computation then shows that the minimum of (A 5) is given by the value $-\alpha_{d}^{2}$ and it is achieved by $x_{N}=\ldots=$ $x_{d-1}=0, x_{d}=\alpha_{d}$. This yields (A 5) and thus concludes the proof for the second inequality in (A 4).

\section{References}

Alberti, G., Choksi, R., \& Otto, F. (2009). Uniform energy distribution for an isoperimetric problem with long-range interactions. J. Amer. Math. Soc., 22(2), 569-605.

Ball, J. M., \& James, R. D. (1987). Fine phase mixtures as minimizers of energy. Arch. Rational Mech. Anal., 100(1), 13-52.

K. Bhattacharya (2003). Microstructure of martensite. Why it forms and how it gives rise to the shape-memory effect. Oxford Series on Materials Modelling. Oxford University Press, Oxford.

Bhattacharya, K. (1993). Comparision of the geometrically nonlinear and linear theories of martensitic transformation. Contin. Mech. Thermodyn., 3, 205-242.

Brener, E., Iordanskii, S., \& Marchenko, V. (1999). Elastic effects on the kinetics of a phase transition. Phys. Rev. Lett., 82(7), 1506-1509.

Capella, A., \& Otto, F. (2009). A rigidity result for a perturbation of the geometrically linear three-well problem. Comm. Pure Appl. Math., 62(12), 1632-1669.

Capella, A., \& Otto, F. (2010). A quantitative rigidity result for the cubic to tetragonal phase transition in the geometrically linear theory with interfacial energy. submitted.

Choksi, R., Conti, S., Kohn, R. V., \& Otto, F. (2008). Ground state energy scaling laws during the onset and destruction of the intermediate state in a type I superconductor. Comm. Pure Appl. Math., 61(5), 595-626.

Choksi, R., \& Kohn, R. V. (1998). Bounds on the micromagnetic energy of a uniaxial ferromagnet. Comm. Pure Appl. Math., 51(3), 259-289.

Choksi, R., Kohn, R. V., \& Otto, F. (1999). Domain branching in uniaxial ferromagnets: a scaling law for the minimum energy. Comm. Math. Phys., 201(1), 61-79.

Dolzmann, G., \& Müller, S. (1995). Microstructures with finite surface energy: the two-well problem. Arch. Rational Mech. Anal., 132(2), 101-141.

Fonseca, I. \& Müller, S. (1991). A uniqueness proof for the Wulff theorem. Proc. Roy. Soc. Edinburgh Sect. A, 119(1-2):125-136.

Khachaturyan, A. (1967). Some questions concerning the theory of phase transformations in solids. Phys. Solid State, 8, 2163-2168.

Khachaturyan, A. (1982). Theory of structural transformations in solids. New York, NY: John Wiley.

Knüpfer, H., \& Muratov, C. (2010). Domain structure of bulk ferromagnetic crystals in applied fields near saturation. Preprint. www.cims.nyu.edu $/{ }^{\sim}$ knuepfer. 
Kohn, R. V. (1991). The relaxation of a double-well energy. Contin. Mech. Thermodyn., 3(3), 193-236.

Kohn, R. V., \& Müller, S. (1992). Branching of twins near an austenite/twinned-martensite interface. Philosophical Magazine A, 66(5), 697-715.

Kohn, R. V., \& Müller, S. (1994). Surface energy and microstructure in coherent phase transitions. Comm. Pure Appl. Math., 47(4), 405-435.

Müller, S., \& Šverák, V. (1996). Attainment results for the two-well problem by convex integration. In Geometric analysis and the calculus of variations, (pp. 239-251). Int. Press, Cambridge, MA.

Mura, T. (1982). Micromechanics of defects in solids. The Hague, Netherlands: Martinus Nijhoff Publishers.

Poduri, R., \& Chen, L. Q. (1996). Non-classical nucleation theory of ordered intermetallic precipitates-application to the Al-Li alloy. Acta Materialia, 44(10), 4253 - 4259.

Roitburd, A. (1969). Domain structure of crystals formed in solid phase. Soviet Phys. Sol. St., 10, 2870.

Taylor, J.E. (1975). Unique structure of solutions to a class of nonelliptic variational problems. Diff. geom. (Proc. Sympos. Pure. Math., Vol. XXVII, Stanford Univ., Stanford, Calif., 1973), Part 1, pages 419-427. Amer. Math. Soc., Providence, R.I..

Voorhees, P. W., McFadden, G. B., \& Johnson, W. C. (1992). On the morphological development of second-phase particles in elastically-stressed solids. Acta Metallurgica et Materialia, 40(11), 2979 - 2992.

Wang, Y. \& Khachaturyan, A. (1994). Effect of antiphase domains on shape and spatial arrangement of coherent ordered intermetallics. Scripta Metallurgica et Materialia, 31(10), $1425-1430$.

Wulff, G. (1901). Zur Frage der Geschwindigkeit des Wachsthums und der Auflösung der Krystallflächen. Zeitschrift für Krystallographie und Mineralogie, 34(5/6):449-530.

Zhang, L., Chen, L.-Q., \& Du, Q. (2007). Morphology of critical nuclei in solid-state phase transformations. Phys. Rev. Lett., 98, 255703.

Zhang, L., Chen, L.-Q., \& Du, Q. (2008). Diffuse-interface description of strain-dominated morphology of critical nuclei in phase transformations. Acta Materialia, 56(14), 35683576 .

Article submitted to Royal Society 